\title{
Strictly $\varphi$-sub-Gaussian quasi shot noise processes
}

\author{
O. I. Vasylyk* \\ Department of Probability Theory, Statistics and Actuarial Mathematics, Faculty of Mechanics and Mathematics, \\ Taras Shevchenko National University of Kyiv, Ukraine
}

(Received: 25 November 2016; Accepted: 5 February 2017)

\begin{abstract}
In the paper, strictly $\varphi$-sub-Gaussian quasi shot noise processes are considered. There are obtained estimates for distribution of supremum of such a process defined on a compact set and formulated conditions for its sample functions continuity with probability one.
\end{abstract}

Keywords Short noise processes, $\varphi$-sub-Gaussian processes.

AMS 2010 subject classifications 60G07, 60G17, 60F10, 60H05, 60K40.

DOI: $10.19139 /$ soic.v5i2.263

\section{Introduction}

Studying properties of distribution of supremum of a stochastic process, problems of existence of moments and exponential moments of suprema, characteristics of sample functions, the behaviour of stochastic processes in different functional spaces are topical subjects in the theory of stochastic processes for many years. Classical results can be found in books by Cramer and Lidbetter (1967) [5], Lidbetter, Lindgren and Rootzen (1983) [28], Adler (1990) [1], Ledoux and Talagrand (1991) [29], Buldygin and Kozachenko (1998) [3], Bulinski and Shiryaev (2005) [4]. A lot of papers contain results on estimation of exponential moments and distribution of suprema of Gaussian processes, in particular, papers by Skorokhod (1970) [32], Landau and Shepp (1970) [27], Ledoux and Talagrand (1991) [29], Liphshits (1995) [30], Yurinsky (1995) [33]. In the end of sixties, there appeared papers, in which wider classes of random variables and processes, than Gaussian one, were studied. In the paper by Kahane (1960) [12] sub-Gaussian random variables were introduced. In 1968 in the paper [13] Kozachenko introduced a notion of sub-Gaussian random process. Properties of such processes were studied, in particular, by Buldygin (1977) [2], Fukuda (1990) [9], Ostrovsky (1991) [31].

In 1985 in the paper by Kozachenko and Ostrovsky [15] there were considered spaces $\operatorname{Sub}_{\varphi}(\Omega)$ of $\varphi$-subGaussian random variables and processes, which appeared to be natural generalization of sub-Gaussian spaces. Buldygin and Kozachenko in [3] presented fundamental properties of random variables and processses from $\operatorname{Sub}_{\varphi}(\Omega)$, conditions for boundedness and estimation of distribution of suprema of such processes in some special cases. Further development of the theory of $\varphi$-sub-Gaussian random processes was presented, in particular, in $[11,16,21,22,24,25]$. Since $\varphi$-sub-Gaussian random processes are more general, than Gaussian and subGaussian processes, they can be used for simulation of real random processes in queueing theory and financial mathematics. In particular, fractional Brownian motion belongs to the $\operatorname{space}_{\operatorname{Sub}}(\Omega)$ with $\varphi(x)=\frac{x^{2}}{2}$. Examples of application and simulation of $\varphi$-sub-Gaussian random processes can be found in $[17,18,19,20]$.

\footnotetext{
${ }^{*}$ Correspondence to: 60 Volodymyrska str., Department of Probability Theory, Statistics and Actuarial Mathematics, Faculty of Mechanics and Mathematics, Taras Shevchenko National University of Kyiv, 01601 Kyiv, Ukraine (ovasylyk@univ.kiev.ua)
}

ISSN 2310-5070 (online) ISSN 2311-004X (print)

Copyright (C) 2017 International Academic Press 
In this paper, some results received for $\varphi$-sub-Gaussian random processes are applied to shot noise processes. Shot noise processes serve as mathematical model of various physical phenomena. They are used in electronics, telecommunications, mesoscopic physics.

In $[3,6]$ there was considered a real-valued homogeneous zero-mean process $\xi=(\xi(t), t \in \mathbb{R})$ with independent increments, defined on a complete probability space $(\Omega, \mathcal{F}, \mathbf{P})$, and a real-valued function $g=(g(t, u), t, u \in \mathbb{R})$ satisfying the condition $\int_{-\infty}^{+\infty} g^{2}(t, u) \mathrm{d} u<\infty, \quad t \in \mathbb{R}$. Then the process $X(t)=\int_{-\infty}^{+\infty} g(t, u) \mathrm{d} \xi(u), \quad t \in \mathbb{R}$, was called a shot noise process generated by the process $\xi$ and the response function $g$. In the papers $[7,8]$ and monographs $[3,6]$ properties of pre-Gaussian shot noise processes were studied and estimates for distribution of suprema for such processes were received.

I shall consider the case, when $\xi=(\xi(t), t \in \mathbb{R})$ is a real-valued zero-mean random process with uncorrelated increments defined on a standard probability space such that

$$
E(\xi(t)-\xi(s))^{2}=t-s, \quad t>s \in \mathbb{R} .
$$

If $\xi$ is a strictly $\varphi$-sub-Gaussian random process, we shall call the process $X(t)=\int_{-\infty}^{+\infty} g(t, u) \mathrm{d} \xi(u)$ strictly $\varphi$-subGaussian quasi shot noise process.

Basic facts and some properties of the $\varphi$-sub-Gaussian random processes are presented in section 1 of this paper. Section 2 contains lemmas, which enable us to consider strictly $\varphi$-sub-Gaussian quasi shot noise processes. In section 3, estimates for distribution of suprema of such a process defined on a compact set are obtained and conditions for its sample functions continuity with probability one are formulated.

\section{Some results from the theory of $\varphi$-sub-Gaussian random processes}

Let's recall some basic facts about the $\operatorname{space}^{\operatorname{Sub}_{\varphi}}(\Omega)$ of generalized sub-Gaussian random variables [3, 11, 25].

\subsection{Space $\operatorname{Sub}_{\varphi}(\Omega)$ of $\varphi$-sub-Gaussian random variables}

Definition 1.1. A continuous even convex function $\varphi=\{\varphi(x), x \in \mathbb{R}\}$ is an Orlicz $N$-function if it is increasing for $x>0, \frac{\varphi(x)}{x} \rightarrow 0$ as $x \rightarrow 0$ and $\frac{\varphi(x)}{x} \rightarrow \infty$ as $x \rightarrow \infty$.

Definition 1.2. The Young-Fenchel transformation $\varphi^{*}$ of an Orlicz N-function $\varphi=\{\varphi(x), x \in \mathbb{R}\}$ is defined as follows

$$
\varphi^{*}(x):=\sup _{y>0}(x y-\varphi(y)), \quad x \geq 0 .
$$

Lemma 1.3. [26] The Young-Fenchel transformation of an Orlicz $N$-function also is $N$-function and the following inequality holds:

$$
x y \leq \varphi(x)+\varphi^{*}(y), \quad \text { where } \quad x>0, y>0 .
$$

Example 1.4. If $\varphi(x)=\frac{|x|^{p}}{p}, p>1$, then $\varphi^{*}(x)=\frac{|x|^{q}}{q}$, where $q$ is such number that $\frac{1}{q}+\frac{1}{p}=1$.

Example 1.5. If $\varphi(x)=\exp \{|x|\}-|x|-1$, then $\varphi^{*}(x)=(|x|+1) \ln (|x|+1)-|x|$.

For more details on properties of convex functions in Orlicz spaces we refer to the book by Krasnosel'skii and Rutickii [26].

Let $(\Omega, \mathcal{F}, \mathbf{P})$ be a standard probability space.

Condition $Q$. We say that condition $Q$ is satisfied, if $\varphi$ is an Orlicz $\mathrm{N}$-function such that

$$
\liminf _{x \rightarrow 0} \frac{\varphi(x)}{x^{2}}=C>0
$$

The constant $C$ may be equal to $+\infty$. 
Definition 1.6. A zero mean random variable $\xi$ belongs to the $\operatorname{space} \operatorname{Sub}_{\varphi}(\Omega)$ (the space of $\varphi$-sub-Gaussian random variables) if there exists a positive constant $a$ such that the inequality

$$
\operatorname{E} \exp (\lambda \xi) \leq \exp (\varphi(a \lambda))
$$

holds for all $\lambda \in \mathbb{R}$.

Example 1.7. The following functions are $\mathrm{N}$-functions satisfying condition Q:

$$
\begin{gathered}
\varphi(x)=\frac{|x|^{\alpha}}{\alpha}, 1<\alpha \leq 2 ; \\
\varphi(x)= \begin{cases}\frac{|x|^{2}}{\alpha}, & |x| \leq 1, \alpha>2 ; \\
\frac{|x|^{\alpha}}{\alpha}, & |x|>1 .\end{cases}
\end{gathered}
$$

Theorem 1.8. [3, 25] The space $\operatorname{Sub}_{\varphi}(\Omega)$ is a Banach space with the norm

$$
\tau_{\varphi}(\xi)=\sup _{\lambda \neq 0} \frac{\varphi^{-1}(\ln E \exp (\lambda \xi))}{|\lambda|}
$$

and the inequalities

$$
\begin{aligned}
E \exp (\lambda \xi) & \leq \exp \left(\varphi\left(\lambda \tau_{\varphi}(\xi)\right)\right) \\
\left(E \xi^{2}\right)^{\frac{1}{2}} & \leq C \tau_{\varphi}(\xi) .
\end{aligned}
$$

hold for all $\lambda \in \mathbb{R}$, where $C>0$ is some constant.

Remark 1.9. If $\varphi(x)=\frac{x^{2}}{2}$, then the space $\operatorname{Sub}_{\varphi}(\Omega)=\operatorname{Sub}(\Omega)$ is called space of sub-Gaussian random variables.

Definition 1.10. Let $(T, \rho)$ be a pseudometric space. The metric entropy is defined as

$$
H(u):=\ln N_{(T, \rho)}(u)
$$

where $N_{(T, \rho)}(u)$ is called a metric massiveness of the set $T$ and denotes the least number of closed $\rho$-balls whose diameter do not exceed $2 u$ needed to cover $T$.

Example 1.11. If $T=[a, b]$ and $\rho$ is the Euclidean distance, then

$$
\ln \left(\max \left\{\frac{b-a}{2 u}, 1\right\}\right) \leq H_{(T, \rho)}(u) \leq \ln \left(\frac{b-a}{2 u}+1\right) .
$$

Definition 1.12. A stochastic process $X=(X(t), t \in T)$ is a $\varphi$-sub-Gaussian process (belongs to the space $\operatorname{Sub}_{\varphi}(\Omega)$ ) if the random variable $X(t) \in \operatorname{Sub}_{\varphi}(\Omega)$ for all $t \in T$.

If $\varphi(x)=\frac{x^{2}}{2}$, then such processes are called sub-Gaussian.

Example 1.13. A centered Gaussian stochastic process is sub-Gaussian process.

\subsection{Strictly $\varphi$-sub-Gaussian random variables and processes}

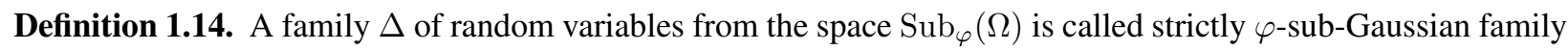
$\left(\Delta \in \operatorname{SSub}_{\varphi}(\Omega)\right)$, if there exists a contstant $C_{\Delta}>0$ such that for any countable set $I, \xi_{i} \in \Delta, i \in I$ and for any $\lambda_{i} \in \mathbb{R}^{1}$ the following inequality holds:

$$
\tau_{\varphi}\left(\sum_{i \in I} \lambda_{i} \xi_{i}\right) \leq C_{\Delta}\left(\mathrm{E}\left(\sum_{i \in I} \lambda_{i} \xi_{i}\right)^{2}\right)^{\frac{1}{2}} .
$$

The constant $C_{\Delta}$ is called identifying constant of the family $\Delta$. 
Remark 1.15. If the set $I$ is countable, then the inequality (1.3) holds true if

$$
\mathrm{E}\left(\sum_{i \in I} \lambda_{i} \xi_{i}\right)^{2}<\infty
$$

Theorem 1.16. [25] Let $\Delta$ be a strictly $\varphi$-sub-Gaussian family of random variables. Then the linear closure of the family $\Delta$ in the space $\operatorname{Sub}_{\varphi}(\Omega)$ (or $L_{2}(\Omega)$ ) also is a strictly $\varphi$-sub-Gaussian family with the same identifying constant.

Definition 1.17. A random process $X=\{X(t), t \in T\}$ is called strictly $\varphi$-sub-Gaussian process (i.e., $X \in$ $\operatorname{SSub}_{\varphi}(\Omega)$ ), if the family of random variables $\{X(t), t \in T\}$ is strictly $\varphi$-sub-Gaussian one. The identifying constant of this family is called identifying constant of the process $X$ and is denoted as $C_{X}$.

Example 1.18. [25] Let $k$ be a deterministic kernel and suppose that the process $X=\{X(t), t \in T\}$ can be represented in the form

$$
X(t)=\int_{T} k(t, s) \mathrm{d} \xi(s),
$$

where $\xi$ is a strictly $\varphi$-sub-Gaussian random process and the integral above is defined in the mean-square sense. Then the process $X$ is strictly $\varphi$-sub-Gaussian random process.

\subsection{Some properties of $\varphi$-sub-Gaussian random processes}

In order to get our main results, we shall use some properties of the $\varphi$-sub-Gaussian random processes obtained by Kozachenko and Vasylyk in [21, 23, 25].

Let $(T, \rho)$ be a pseudometric (metric) separable space with a pseudometric (metric) $\rho$, and $X=\{X(t), t \in T\}$ be a $\varphi$-sub-Gaussian random process.

Suppose that there exists a continuous monotonically increasing function $\sigma=\{\sigma(h), h>0\}$, such that $\sigma(h) \rightarrow 0$ as $h \rightarrow 0$ and

$$
\sup _{\rho(t, s) \leq h} \tau_{\varphi}(X(t)-X(s)) \leq \sigma(h) .
$$

In particular, the following function has this property

$$
\sigma(h)=\sup _{\rho(t, s) \leq h} \tau_{\varphi}(X(t)-X(s)),
$$

if the process $X$ is continuous in the norm $\tau_{\varphi}(\cdot)$.

Consider a compact set $B \subset T$, which consists of at least two points $t$ and $s$ such that $\rho(t, s)>0$. In what follows, we shall use the next notations:

- $\gamma=\sup _{t \in B} \tau_{\varphi}(X(t))$,

- $\beta>0$ is a number such that $\beta \leq \sigma\left(\inf _{s \in B} \sup _{t \in B} \rho(t, s)\right)$,

- $\zeta_{\varphi}(v)=\frac{v}{\varphi^{(-1)}(v)}$.

- $H_{B}(u)=H_{(B, \rho)}(u)$ is metric entropy of the space $(B, \rho)$.

Theorem 1.19. [21, 25] Let $X=\{X(t), t \in B\}$ be a $\varphi$-sub-Gaussian random process satisfying the condition (1.4). If

$$
\int_{0}^{\beta} \zeta_{\varphi}\left(H_{B}\left(\sigma^{(-1)}(u)\right)\right) d u<\infty
$$


then for all $x>D(p, \gamma, \beta)$ and any $p \in(0,1)$, we have the following estimates:

$$
\begin{aligned}
& \mathbf{P}\left\{\sup _{t \in B} X(t)>x\right\} \leq W(p, x), \\
& \mathbf{P}\left\{\inf _{t \in B} X(t)<-x\right\} \leq W(p, x), \\
& \mathbf{P}\left\{\sup _{t \in B}|X(t)|>x\right\} \leq 2 W(p, x),
\end{aligned}
$$

where

$$
\begin{gathered}
W(p, x)=\exp \left\{-Z_{p, \gamma, \beta}^{*}(x-D(p, \gamma, \beta))\right\}, \\
D(p, \gamma, \beta)=2\left(\gamma \zeta_{\varphi}\left(\boldsymbol{H}_{B}\left(\sigma^{(-1)}(p \beta)\right)\right)+\frac{1}{(1-p) p} \int_{0}^{\beta p^{2}} \zeta_{\varphi}\left(\boldsymbol{H}_{B}\left(\sigma^{(-1)}(u)\right)\right) d u\right),
\end{gathered}
$$

$Z_{p, \gamma, \beta}^{*}$ is the Young-Fenchel transformation of the function

$$
Z_{p, \gamma, \beta}(\lambda)=\varphi\left(\frac{\lambda \gamma}{1-p}\right)(1-p)+\varphi\left(\frac{\lambda \beta}{1-p}\right) p, \quad \lambda \in \mathbb{R}
$$

Theorem 1.20. [23, 25] If for a separable $\varphi$-sub-Gaussian random process $X=\{X(t), t \in B\}$ condition (1.4) holds and for arbitrary $\varepsilon>0$

$$
\int_{0}^{\sigma(\varepsilon)} \zeta_{\varphi}\left(H_{B}\left(\sigma^{(-1)}(u)\right)\right) d u<\infty
$$

then the process $X$ is sample continuous process with probability one and for any $x>B(p, \varepsilon)$ we have the following estimate:

$$
\mathbf{P}\left\{\sup _{\rho(t, s)<\varepsilon}|X(t)-X(s)|>x\right\} \leq 2 \exp \left\{-\varphi^{*}\left(\frac{x-B(p, \varepsilon)}{A(p, \varepsilon)}\right)\right\},
$$

where $p \in(0,1), A(p, \varepsilon)=\frac{\sigma(\varepsilon)(3-p)}{(1-p)^{2}}, B(p, \varepsilon)=\frac{4(3-p)}{3 p(1-p)^{2}} \int_{0}^{\sigma(\varepsilon)} \zeta_{\varphi}\left(H_{B}\left(\sigma^{(-1)}(u)\right)\right) d u$.

\section{Strictly $\varphi$-sub-Gaussian quasi shot noise processes}

Let $\xi=(\xi(t), t \in \mathbb{R})$ be a real-valued zero-mean random process with uncorrelated increments defined on a standard probability space such that

$$
E(\xi(t)-\xi(s))^{2}=t-s, \quad t>s \in \mathbb{R} .
$$

Let $g=(g(t, u), t, u \in \mathbb{R})$ be a real-valued function satisfying the following condition:

$$
\int_{-\infty}^{+\infty} g^{2}(t, u) \mathrm{d} u<\infty, \quad t \in \mathbb{R} .
$$

Definition 2.1. We shall call the process

$$
X(t)=\int_{-\infty}^{+\infty} g(t, u) \mathrm{d} \xi(u), \quad t \in \mathbb{R},
$$

a quasi shot noise process generated by the process $\xi$ and the response function $g$, where the integral in (2.2) is defined in the mean-square sense. 
The covariance function of the process $X$ has the following form:

$$
\mathrm{E} X(t) X(s)=\int_{-\infty}^{+\infty} g(t, u) g(s, u) \mathrm{d} u, \quad t, s \in \mathbb{R} .
$$

The following lemma will allow us to introduce a strictly $\varphi$-sub-Gaussian quasi shot noise process.

Lemma 2.2. Let the process $\xi=(\xi(t), t \in \mathbb{R})$ be strictly $\varphi$-sub-Gaussian random process with uncorrelated increments.

Then the process $X(t)=\int_{-\infty}^{+\infty} g(t, u) d \xi(u), t \in \mathbb{R}$, also is strictly $\varphi$-sub-Gaussian process and for any $t, s \in \mathbb{R}$

$$
\begin{array}{r}
\tau_{\varphi}(X(t)) \leq c_{\xi}\left(\int_{-\infty}^{+\infty} g^{2}(t, u) d u\right)^{1 / 2}, \\
\tau_{\varphi}(X(t)-X(s)) \leq c_{\xi}\left(\int_{-\infty}^{+\infty}(g(t, u)-g(s, u))^{2} d u\right)^{1 / 2},
\end{array}
$$

where $c_{\xi}$ is the identifying constant of the process $\xi$.

Proof

From theorem 1.16 and example 1.18 follows that the process $X(t)=\int_{-\infty}^{+\infty} g(t, u) \mathrm{d} \xi(u), t \in \mathbb{R}$, is strictly $\varphi$-subGaussian process with the identifying constant $c_{\xi}$. Therefore, from the definition of strictly $\varphi$-sub-Gaussian random process follows that for any $t \in \mathbb{R}$

$$
\begin{gathered}
\tau_{\varphi}(X(t)) \leq c_{\xi}\left(\mathrm{E} X^{2}(t)\right)^{1 / 2}=c_{\xi}\left(\int_{-\infty}^{+\infty} g^{2}(t, u) \mathrm{d} u\right)^{1 / 2}, \\
\tau_{\varphi}(X(t)-X(s)) \leq c_{\xi}\left(\mathrm{E}(X(t)-X(s))^{2}\right)^{\frac{1}{2}}=c_{\xi}\left(\int_{-\infty}^{+\infty}(g(t, u)-g(s, u))^{2} \mathrm{~d} u\right)^{\frac{1}{2}} .
\end{gathered}
$$

Lemma 2.3. Let $X(t)=\int_{-\infty}^{+\infty} g(t, u) d \xi(u), t \in \mathbb{R}$, be strictly $\varphi$-sub-Gaussian process and $\{g(t, u), t, u \in \mathbb{R}\}$ be a complete system of functions in $L^{2}(\mathbb{R})$. Then the process $\xi=(\xi(t), t \in \mathbb{R})$ is strictly $\varphi$-sub-Gaussian random process.

Proof

If the process $X$ can be presented in the form (2.2), then its covariance function has the form $\mathrm{E} X(t) X(s)=$ $\int_{-\infty}^{+\infty} g(t, u) g(s, u) \mathrm{d} u, \quad t, s \in \mathbb{R}$. According to the Karhunen theorem $[4,10]$, the process $\xi=(\xi(t), t \in \mathbb{R})$ belongs to the linear span $H_{X}$ of the values $X(t), t \in \mathbb{R}$ if and only if the system of functions $\{g(t, u), t, u \in \mathbb{R}\}$ is a complete system of functions in $L^{2}(\mathbb{R})$. In our case, linear span $H_{X}$ is a space of strictly $\varphi$-sub-Gaussian random variables. Thus, if $\{g(t, u), t, u \in \mathbb{R}\}$ is complete system of functions in $L^{2}(\mathbb{R})$, then the process $\xi=(\xi(t), t \in \mathbb{R})$ is a strictly $\varphi$-sub-Gaussian random process.

Definition 2.4. We shall call the process $X(t)=\int_{-\infty}^{+\infty} g(t, u) \mathrm{d} \xi(u)$ a strictly $\varphi$-sub-Gaussian quasi shot noise process, if $\xi$ is strictly $\varphi$-sub-Gaussian random process.

\section{Main results}

Suppose that there exist such functions $r=(r(h), h \geq 0)$ and $k=(k(u), u \in \mathbb{R})$ that

$$
|g(t, u)-g(s, u)| \leq r(t-s) k(u), \quad t, s, u \in \mathbb{R},
$$

the function $r$ is nonnegative continuous monotonically increasing function, such that $r(h) \rightarrow 0$ as $h \rightarrow 0$, and the function $k$ is nonnegative continuous function that satisfies the condition $\int_{\mathbb{R}} k^{2}(u) \mathrm{d} u<\infty$. 
Theorem 3.1. Let $X$ be a strictly $\varphi$-sub-Gaussian quasi shot noise process on the interval $[a, b], a, b \in \mathbb{R}$, with the response function $g$ satisfying condition (3.1). If

$$
\int_{0}^{\beta} \zeta_{\varphi}\left(\ln \left(\frac{b-a}{2 r^{(-1)}(c u)}+1\right)\right) d u<\infty,
$$

then for all $x>\bar{D}(p, \gamma, \beta)$ and any $p \in(0,1)$, we have the following estimates:

$$
\begin{aligned}
& \mathbf{P}\left\{\sup _{t \in[a, b]} X(t)>x\right\} \leq \bar{W}(p, x), \\
& \mathbf{P}\left\{\inf _{t \in[a, b]} X(t)<-x\right\} \leq \bar{W}(p, x), \\
& \mathbf{P}\left\{\sup _{t \in[a, b]}|X(t)|>x\right\} \leq 2 \bar{W}(p, x),
\end{aligned}
$$

where

$$
\begin{gathered}
\bar{W}(p, x)=\exp \left\{-Z_{p, \gamma, \beta}^{*}(x-\bar{D}(p, \gamma, \beta))\right\}, \\
\bar{D}(p, \gamma, \beta)=2\left(\gamma \zeta_{\varphi}\left(\ln \left(\frac{b-a}{2 r^{(-1)}(c p \beta)}+1\right)\right)+\right. \\
\left.\frac{1}{(1-p) p} \int_{0}^{\beta p^{2}} \zeta_{\varphi}\left(\ln \left(\frac{b-a}{2 r^{(-1)}(c u)}+1\right)\right) d u\right),
\end{gathered}
$$

$Z_{p, \gamma, \beta}^{*}$ is the Young-Fenchel transformation of the function

$$
\begin{gathered}
Z_{p, \gamma, \beta}(\lambda)=\varphi\left(\frac{\lambda \gamma}{1-p}\right)(1-p)+\varphi\left(\frac{\lambda \beta}{1-p}\right) p, \quad \lambda \in \mathbb{R}, \\
\gamma=\sup _{t \in[a, b]} c_{\xi}\left(\int_{-\infty}^{+\infty} g^{2}(t, u) d u\right)^{1 / 2}, \beta \in\left(0, \frac{1}{c} r\left(\frac{b-a}{2}\right)\right], c=\left(c_{\xi}\left(\int_{-\infty}^{+\infty} k^{2}(u) d u\right)^{1 / 2}\right)^{-1} .
\end{gathered}
$$

Proof

If condition (3.1) holds for the response function $g$, then from lemma 2.2 we have that for $t, s \in[a, b]$

$$
\begin{gathered}
\tau_{\varphi}(X(t)-X(s)) \leq c_{\xi}\left(\int_{-\infty}^{+\infty}(g(t, u)-g(s, u))^{2} \mathrm{~d} u\right)^{1 / 2} \leq \\
c_{\xi}\left(\int_{-\infty}^{+\infty}(r(t-s) k(u))^{2} \mathrm{~d} u\right)^{1 / 2}=c_{\xi} r(t-s)\left(\int_{-\infty}^{+\infty} k^{2}(u) \mathrm{d} u\right)^{1 / 2},
\end{gathered}
$$

where the function $r=(r(h), h \geq 0)$ is nonnegative continuous monotonically increasing function, such that $r(h) \rightarrow 0$ as $h \rightarrow 0$, and the function $k=(k(u), u \in \mathbb{R})$ is nonnegative and satisfies the condition $\int_{\mathbb{R}} k^{2}(u) \mathrm{d} u<\infty$.

This means that there exists a continuous monotonically increasing function

$$
\sigma(h)=c_{\xi} r(h)\left(\int_{-\infty}^{+\infty} k^{2}(u) \mathrm{d} u\right)^{1 / 2}, \quad h \geq 0,
$$

such that $\sigma(h) \rightarrow 0$ as $h \rightarrow 0$ and for which the condition (1.4) holds true:

$$
\sup _{|t-s| \leq h} \tau_{\varphi}(X(t)-X(s)) \leq \sigma(h) .
$$


Under condition (1.5) of theorem 1.19, for $B=[a, b]$ we get that $\sigma^{(-1)}(u)=r^{(-1)}(c u)$,

$$
H_{B}\left(\sigma^{(-1)}(u)\right) \leq \ln \left(\frac{b-a}{2 \sigma^{(-1)}(u)}+1\right)=\ln \left(\frac{b-a}{2 r^{(-1)}(c u)}+1\right)
$$

where $c=\left(c_{\xi}\left(\int_{-\infty}^{+\infty} k^{2}(u) \mathrm{d} u\right)^{1 / 2}\right)^{-1}$.

Since $\zeta_{\varphi}(v)=\frac{v}{\varphi^{(-1)}(v)}$ is continuous monotonically increasing function, then

$$
\begin{gathered}
\int_{0}^{\beta} \zeta_{\varphi}\left(H_{B}\left(\sigma^{(-1)}(u)\right)\right) \mathrm{d} u \leq \int_{0}^{\beta} \zeta_{\varphi}\left(\ln \left(\frac{b-a}{2 r^{(-1)}(c u)}+1\right)\right) \mathrm{d} u, \\
D(p, \gamma, \beta)=2\left(\gamma \zeta_{\varphi}\left(H_{B}\left(\sigma^{(-1)}(p \beta)\right)\right)+\frac{1}{(1-p) p} \int_{0}^{\beta p^{2}} \zeta_{\varphi}\left(H_{B}\left(\sigma^{(-1)}(u)\right)\right) d u\right) \leq \\
2\left(\gamma \zeta_{\varphi}\left(\ln \left(\frac{b-a}{2 r^{(-1)}(c p \beta)}+1\right)\right)+\right. \\
\left.\frac{1}{(1-p) p} \int_{0}^{\beta p^{2}} \zeta_{\varphi}\left(\ln \left(\frac{b-a}{2 r^{(-1)}(c u)}+1\right)\right) d u\right)=: \bar{D}(p, \gamma, \beta),
\end{gathered}
$$

where $\gamma=\sup _{t \in[a, b]} c_{\xi}\left(\int_{-\infty}^{+\infty} g^{2}(t, u) \mathrm{d} u\right)^{1 / 2}, \beta \in\left(0, c_{\xi} r\left(\frac{b-a}{2}\right)\left(\int_{-\infty}^{+\infty} k^{2}(u) \mathrm{d} u\right)^{1 / 2}\right]=\left(0, \frac{1}{c} r\left(\frac{b-a}{2}\right)\right]$.

Thus, if condition (3.2) holds, then the assertion of this theorem follows from theorem 1.19.

Theorem 3.2. If for a separable strictly $\varphi$-sub-Gaussian quasi shot noise process $X=\{X(t), t \in[a, b]\}$ condition (3.1) holds and for arbitrary $\varepsilon>0$

$$
\int_{0}^{\sigma(\varepsilon)} \zeta_{\varphi}\left(\ln \left(\frac{b-a}{2 r^{(-1)}(c u)}+1\right)\right) d u<\infty
$$

then the process $X$ is a sample continuous process with probability one and for any $p \in(0,1)$ and $x>\bar{B}(p, \varepsilon)$ we have the following estimate:

$$
\mathbf{P}\left\{\sup _{|t-s|<\varepsilon}|X(t)-X(s)|>x\right\} \leq 2 \exp \left\{-\varphi^{*}\left(\frac{x-\bar{B}(p, \varepsilon)}{\bar{A}(p, \varepsilon)}\right)\right\},
$$

where $\sigma(\varepsilon)=c_{\xi} r(\varepsilon)\left(\int_{-\infty}^{+\infty} k^{2}(u) d u\right)^{1 / 2}, \bar{A}(p, \varepsilon)=\frac{\sigma(\varepsilon)(3-p)}{(1-p)^{2}}$,

$\bar{B}(p, \varepsilon)=\frac{4(3-p)}{3 p(1-p)^{2}} \int_{0}^{\sigma(\varepsilon)} \zeta_{\varphi}\left(\ln \left(\frac{b-a}{2 r^{(-1)}(c u)}+1\right)\right) d u, c=c_{\xi}^{-1}\left(\int_{-\infty}^{+\infty} k^{2}(u) d u\right)^{-1 / 2}$.

Proof

Consider a separable strictly $\varphi$-sub-Gaussian quasi shot noise process $X=\{X(t), t \in[a, b]\}$ and let condition (3.1) hold. Then there exists the function

$$
\sigma(h)=c_{\xi} r(h)\left(\int_{-\infty}^{+\infty} k^{2}(u) \mathrm{d} u\right)^{1 / 2}, \quad h \geq 0,
$$


for which condition (1.4) holds true.

From (3.3) we get fulfilment of condition (1.6) of theorem 1.20:

$$
\begin{gathered}
H_{B}\left(\sigma^{(-1)}(u)\right) \leq \ln \left(\frac{b-a}{2 \sigma^{(-1)}(u)}+1\right)=\ln \left(\frac{b-a}{2 r^{(-1)}(c u)}+1\right), \\
\int_{0}^{\sigma(\varepsilon)} \zeta_{\varphi}\left(H_{B}\left(\sigma^{(-1)}(u)\right)\right) d u \leq \int_{0}^{\sigma(\varepsilon)} \zeta_{\varphi}\left(\ln \left(\frac{b-a}{2 r^{(-1)}(c u)}+1\right)\right) d u<\infty,
\end{gathered}
$$

where $\sigma(\varepsilon)=c_{\xi} r(\varepsilon)\left(\int_{-\infty}^{+\infty} k^{2}(u) \mathrm{d} u\right)^{1 / 2}$, and $c=c_{\xi}^{-1}\left(\int_{-\infty}^{+\infty} k^{2}(u) \mathrm{d} u\right)^{-1 / 2}$

This implies that the considered separable strictly $\varphi$-sub-Gaussian quasi shot noise process $X$ satisfies conditions of theorem 1.20 and from (1.7) estimate (3.4) follows. For $\bar{B}(p, \varepsilon)$ from estimate (3.4) in this particular case we get the following expression:

$$
\bar{B}(p, \varepsilon)=\frac{4(3-p)}{3 p(1-p)^{2}} \int_{0}^{\sigma(\varepsilon)} \zeta_{\varphi}\left(\ln \left(\frac{b-a}{2 r^{(-1)}(c u)}+1\right)\right) \mathrm{d} u .
$$

Example 3.3. Let's illustrate theorem 3.1 by the following example.

Consider a zero-mean random process $\xi=(\xi(t), t \in \mathbb{R}) \in \operatorname{SSub}_{\varphi}(\Omega)$ with uncorrelated increments, such that $\varphi(x)=\frac{x^{2}}{2}, x \in \mathbb{R}$. That is, $\xi$ is a strictly sub-Gaussian process and $\mathbf{E} \xi^{2}(t)=\tau^{2}(\xi(t))$ for all $t \in \mathbb{R}$, where $\tau(\cdot)=\tau_{\varphi}(\cdot)$ is a norm in the space of sub-Gaussian variables $\operatorname{Sub}(\Omega)$ (see [3]).

In this case we have the identifying constant $c_{\xi}=1$, the inverse function $\varphi^{(-1)}(v)=\sqrt{2 v}, v \geq 0$, and $\zeta_{\varphi}(v)=\frac{v}{\varphi^{(-1)}(v)}=\frac{v}{\sqrt{2 v}}=\sqrt{\frac{v}{2}}$.

Let a real-valued function $g=(g(t, u), t, u \in \mathbb{R})$ have the following form:

$$
g(t, u)=\frac{\sin (t u)}{|u|^{\delta}+1}, \quad t, u \in \mathbb{R}, \quad \delta>1 .
$$

This function satisfies the condition:

$$
\int_{-\infty}^{+\infty} g^{2}(t, u) \mathrm{d} u=\int_{-\infty}^{+\infty} \frac{\sin ^{2}(t u)}{\left(|u|^{\delta}+1\right)^{2}} \mathrm{~d} u<\infty, \quad t \in \mathbb{R}
$$

Now, we can consider a strictly sub-Gaussian quasi shot noise process $X(t)=\int_{-\infty}^{+\infty} g(t, u) \mathrm{d} \xi(u), \quad t \in \mathbb{R}$, generated by the process $\xi$ and the response function $g$.

For the function $g$ we get the following estimate:

$$
\begin{gathered}
|g(t, u)-g(s, u)|=\frac{|\sin (t u)-\sin (s u)|}{|u|^{\delta}+1}=\frac{\left|2 \sin \frac{u(t-s)}{2} \cos \frac{u(t-s)}{2}\right|}{|u|^{\delta}+1} \leq \\
2\left|\frac{u(t-s)}{2}\right|^{\alpha} \frac{1}{|u|^{\delta}+1}=\frac{2^{1-\alpha}|u|^{\alpha}|t-s|^{\alpha}}{|u|^{\delta}+1}=|t-s|^{\alpha} \cdot \frac{2^{1-\alpha}|u|^{\alpha}}{|u|^{\delta}+1}=r(t-s) k(u),
\end{gathered}
$$

where

$$
r(t-s)=|t-s|^{\alpha}, \quad k(u)=\frac{2^{1-\alpha}|u|^{\alpha}}{|u|^{\delta}+1}, \quad t, s, u \in \mathbb{R}, \delta>1, \alpha=\min (1, \delta-1) \in(0,1] .
$$

The function $r(h)=|h|^{\alpha}, h \in \mathbb{R}$, is a nonnegative continuous monotonically increasing function such that $r(h) \rightarrow 0$ as $h \rightarrow 0$. 
The function $k(u)=\frac{2^{1-\alpha}|u|^{\alpha}}{|u|^{\delta}+1}, u \in \mathbb{R}$, is a nonnegative continuous function and

$$
\int_{\mathbb{R}} k^{2}(u) \mathrm{d} u=\int_{\mathbb{R}} \frac{2^{2-2 \alpha}|u|^{2 \alpha}}{\left(|u|^{\delta}+1\right)^{2}} \mathrm{~d} u<\infty .
$$

This means that condition (3.1) holds for the function $g$. This implies that in our case there exists a continuous monotonically increasing function

$$
\sigma(h)=c_{\xi} r(h)\left(\int_{-\infty}^{+\infty} k^{2}(u) \mathrm{d} u\right)^{1 / 2}=h^{\alpha}\left(\int_{\mathbb{R}} \frac{2^{2-2 \alpha}|u|^{2 \alpha}}{\left(|u|^{\delta}+1\right)^{2}} \mathrm{~d} u\right)^{1 / 2}, \quad h \geq 0,
$$

such that $\sigma(h) \rightarrow 0$ as $h \rightarrow 0$ and for which the condition (1.4) holds true.

Consider the process $X$ on the interval $[a, b] \subset \mathbb{R}$. For functions in condition (3.2) we obtain the following expressions:

$$
\begin{gathered}
\ln \left(\frac{b-a}{2 r^{(-1)}(c u)}+1\right)=\ln \left(\frac{b-a}{2(c u)^{1 / \alpha}}+1\right) \\
\zeta_{\varphi}\left(\ln \left(\frac{b-a}{2 r^{(-1)}(c u)}+1\right)\right) \mathrm{d} u=\frac{\ln \left(\frac{b-a}{2(c u)^{1 / \alpha}}+1\right)}{\left(2 \ln \left(\frac{b-a}{2(c u)^{1 / \alpha}}+1\right)\right)^{1 / 2}}=\left(\frac{1}{2} \ln \left(\frac{b-a}{2(c u)^{1 / \alpha}}+1\right)\right)^{1 / 2},
\end{gathered}
$$

where $c=\left(\int_{-\infty}^{+\infty} k^{2}(u) \mathrm{d} u\right)^{-\frac{1}{2}}$.

In this case condition (3.2) is of the form

$$
\int_{0}^{\beta} \zeta_{\varphi}\left(\ln \left(\frac{b-a}{2 r^{(-1)}(c u)}+1\right)\right) \mathrm{d} u=\int_{0}^{\beta}\left(\frac{1}{2} \ln \left(\frac{b-a}{2(c u)^{1 / \alpha}}+1\right)\right)^{1 / 2} \mathrm{~d} u<\infty
$$

where $\beta \in\left(0, \frac{1}{c}\left(\frac{b-a}{2}\right)^{\alpha}\right]$. Since the integral above converges, the strictly sub-Gaussian quasi shot noise process $X$ generated by the process $\xi$ and the response function $g$ satisfies conditions of theorem 3.1.

Now consider functions in the expression $\bar{W}(p, x)=\exp \left\{-Z_{p, \gamma, \beta}^{*}(x-\bar{D}(p, \gamma, \beta))\right\}$.

For $\bar{D}(p, \gamma, \beta)$ we get the expression

$$
\bar{D}(p, \gamma, \beta)=2\left(\gamma\left(\frac{1}{2} \ln \left(\frac{b-a}{2(c p \beta)^{1 / \alpha}}+1\right)\right)^{1 / 2}+\frac{1}{(1-p) p} \int_{0}^{\beta p^{2}}\left(\frac{1}{2} \ln \left(\frac{b-a}{2(c u)^{1 / \alpha}}+1\right)\right)^{1 / 2} \mathrm{~d} u\right) .
$$

Recall, that here $p \in(0,1)$ and $\gamma=\sup _{t \in[a, b]} c_{\xi}\left(\int_{-\infty}^{+\infty} g^{2}(t, u) \mathrm{d} u\right)^{1 / 2}=\sup _{t \in[a, b]}\left(\int_{-\infty}^{+\infty} \frac{\sin ^{2}(t u)}{\left(|u|^{\delta}+1\right)^{2}} \mathrm{~d} u\right)^{1 / 2}$.

The function $Z_{p, \gamma, \beta}(\lambda)$ will take the form

$$
Z_{p, \gamma, \beta}(\lambda)=\frac{1}{2}\left(\frac{\lambda \gamma}{1-p}\right)^{2}(1-p)+\frac{1}{2}\left(\frac{\lambda \beta}{1-p}\right)^{2} p=\frac{\lambda^{2}\left(\gamma^{2}(1-p)+\beta^{2} p\right)}{2(1-p)^{2}}, \quad \lambda \in \mathbb{R},
$$

and for its Young-Fenchel transformation we have

$$
Z_{p, \gamma, \beta}^{*}(x-\bar{D}(p, \gamma, \beta))=\frac{(x-\bar{D}(p, \gamma, \beta))^{2}(1-p)^{2}}{2\left(\gamma^{2}(1-p)+\beta^{2} p\right)}, \quad x>\bar{D}(p, \gamma, \beta) .
$$

Thus,

$$
\bar{W}(p, x)=\exp \left\{-Z_{p, \gamma, \beta}^{*}(x-\bar{D}(p, \gamma, \beta))\right\}=\exp \left\{-\frac{(x-\bar{D}(p, \gamma, \beta))^{2}(1-p)^{2}}{2\left(\gamma^{2}(1-p)+\beta^{2} p\right)}\right\}
$$


and, finally, for all $x>\bar{D}(p, \gamma, \beta)$ and any $p \in(0,1)$ we get the following estimate for distribution of supremum of the process $X$ on the interval $[a, b]$ :

$$
\mathbf{P}\left\{\sup _{t \in[a, b]} X(t)>x\right\} \leq \exp \left\{-\frac{(x-\bar{D}(p, \gamma, \beta))^{2}(1-p)^{2}}{2\left(\gamma^{2}(1-p)+\beta^{2} p\right)}\right\}
$$

One can see that the right part of the estimate above tends to zero as $x \rightarrow \infty$.

\section{Conclusions}

In this paper, strictly $\varphi$-sub-Gaussian quasi shot noise processes are introduced. Some results, obtained for $\varphi$-subGaussian stochastics processes, are applied to strictly $\varphi$-sub-Gaussian quasi shot noise processes. Estimates for distribution of supremum and conditions for sample functions continuity with probability one for such processes defined on a compact set are presented. As an example, strictly sub-Gaussian quasi shot noise processes are considered.

\section{Acknowledgments}

I am very grateful to referees for comments, which helped me to improve results of this paper. Special thanks to Professor Yuriy Kozachenko for encouragement and valuable advice.

\section{REFERENCES}

1. R.J. Adler, An introduction to continuity, extrema and related topics for general Gaussian processes, Lecture Notes-Monograph Series. Institute of Mathematical Statistics, Hayward, Vol. 12, 1990.

2. V.V. Buldygin, Sub-Gaussian processes and convergencs of random series in functional spaces, Ukrainian Mathematical Journal, Vol. 29, No. 4, pp. 443-454, 1977.

3. V. V. Buldygin, and Yu. V. Kozachenko, Metric Characterization of Random Variables and Random Processes, American Mathematical Society, Providence, RI, 2000.

4. A.V. Bulinski, and A.N. Shiryaev, Theory of Stochastic Processes, Moscow: "Fizmatlit", 408 p., 2005. (In Russian)

5. H. Cramer, and M.R. Leadbetter, Stationary and related stochastic processes. Sample function properties and their applications, Wiley, New York-London-Sydney, 348 p., 1967.

6. I.V. Dariychuk, Yu.V. Kozachenko, and M.M. Perestyuk, Stochastic processes from Orlicz spaces, Chernivtsi: "Zoloti lytavry", 212 p., 2011. (In Ukrainian)

7. I.V. Dariychuk, and Yu.V. Kozachenko, Some properties of pre-Gaussian shot noise processes, Stochastic Analysis and Random Dynamics. International Conference. Abstracts, Lviv, Ukraine, pp. 57-59, 2009.

8. I.V. Dariychuk, Yu.V. Kozachenko, The distribution of the supremum of $\Theta$-pre-Gaussian shot noise processes, Theory of Probability and Mathematical Statistics), No. 80, pp. 85-100, 2010.

9. R. Fukuda, Exponential integrability of sub-Gaussian vectors, Probab. Theory Related Fields, Vol. 85, No. 4, pp. 505-521, 1990.

10. I.I. Gikhman, and A.V. Skorokhod, Theory of random processes, Vol.1. Moscow: "Nauka", 1971; English trans., Springer-Verlag, Berlin-Heidelberg-New York, 1974.

11. R. Giuliano Antonini, Yu. V. Kozachenko, T. Nikitina, Space of $\varphi$-sub-Gaussian random variables, Rend. Accad. Naz. Sci. XL Mem. Mat. Appl. (5) 27, pp. 92-124, 2003

12. J.P. Kahane, Propriétés locales des fonctions à series de Fouries aléatories, Studia Math., Vol. 19, No. 1, pp. 1-25, 1960.

13. Yu. Kozachenko, Sufficient conditions for continuity with probability one of sub-Gaussian stochastic processes, Dopovidi AN URSR, No. 2, pp. 113-115, 1968

14. Yu. Kozachenko, Random processes in Orlicz spaces. I. Probability Theory and Mathematical Statistics, No. 30, pp.103-117, 1984 Random processes in Orlicz spaces. II. Probability Theory and Mathematical Statistics, No. 31, pp.51-58, 1984.

15. Yu. Kozachenko, E. Ostrovsky, Banach spaces of random variables of sub-Gaussian type, Theory of Probability and Mathematical Statistics, No. 32, pp.42-53, 1985.

16. Yu. Kozachenko, M. Perestyuk, O. Vasylyk, On Uniform Convergence of Wavelet Expansions of $\varphi$-sub-Gaussian Random Process, Random Operators and Stochastic Equations, Vol.14, No.3, pp. 209-232, 2006.

17. Yu. Kozachenko, I. Rozora, Accuracy and Reliability of models of stochastic processes of the space $\operatorname{Sub}_{\varphi}(\Omega)$, Theory of Probability and Mathematical Statistics, No. 71, pp. 105-117, 2005.

18. Yu. Kozachenko, T. Sottinen, O. Vasilik, Self-similar processes with stationary increments in the spaces $S_{S u b}(\Omega)$, Theory of Probability and Mathematical Statistics, No. 65, pp.77-88, 2002. 
19. Yu. Kozachenko, T. Sottinen, O. Vasylyk, Path space large deviations of a large buffer with Gaussian input traffic, Queueing Systems, No. 42, pp.113-129, 2002.

20. Yu. Kozachenko, T. Sottinen, O. Vasylyk, Simulation of Weakly Self-Similar Stationary Increment Sub $\varphi(\Omega)$-Processes: A Series Expansion Approach, Methodology and Computing in Applied Probability, Vol. 7, No. 3, pp. 379-400, 2005.

21. Yu. Kozachenko, O. Vasilik, On the distribution of suprema of $\operatorname{Sub}_{\varphi}(\Omega)$ random processes, Theory of Stochastic Processes, Vol. 4(20), issue 1-2, pp. 147-160, 1998.

22. Yu. Kozachenko, O. Vasilik, Stochastic processes of the classes $V(\varphi, \psi)$, Theory of Probability and Mathematical Statistics, No. 63, pp.109-121, 2001.

23. Yu. Kozachenko, O. Vasylyk, Sample pathes continuity and estimates of distributions of the increments of separable stochastic processes from the class $V(\varphi, \psi)$, defined on a compact set, Bulletin of the University of Kiev, Series: Physics and Mathematics, Issue 2, pp.45-50, 2004. (In Ukrainian)

24. Yu. Kozachenko, R. Yamnenko, O. Vasylyk, Upper estimate of overrunning by $\operatorname{Sub}_{\varphi}(\Omega)$ random process the level specified by continuous function, Random Oper. Stoch. Equ., Vol. 13, No. 2, pp. 111-128, 2005.

25. Yu. Kozachenko, R. Yamnenko, O. Vasylyk, $\varphi$-sub-Gaussian random process, Kyiv: Vydavnycho-Poligrafichnyi Tsentr, Kyivskyi Universytet (ISBN 978-966-439-051-1), 231 p., 2008. (In Ukrainian).

26. M.A. Krasnosel'skii, Ya.B. Rutickii, Convex Functions and Orlicz Spaces. Moscow, 1958 (in Russian). English translation: P.Noordhoff Ltd, Groningen, 1961, 249p.

27. H.J. Landau, L.A. Shepp, On the supremum of Gaussian processes, Sankhya: The Indian Journal of Statistics, Series A, Vol. 32, No. 4, pp. 369-378, 1970.

28. M.R. Leadbetter, G. Lindgren, H. Rootzen, Extremes and related properties of random sequences and processes, Springer, Berlin, 1983.

29. M. Ledoux, M. Talagrand, Probability in Banach space, Springer-Verlag, Berlin-New York, 480 p., 1991.

30. V.A. Liphshits, Gaussian random functions, Kiev: TViMS, 246 p., 1995.

31. E. I. Ostrovsky, Exponential Bounds for the Distribution of the Maximum of a Non-Gaussian Random Field, Theory of probability and its applications, Vol. 35, No. 3, pp. 487-499, 1991.

32. A.V. Skorokhod, A Note on Gaussian Measures in a Banach Space, Theory of probability and its applications, Vol. 15, No. 3, pp. 519-520, 1970

33. V. Yurinsky, Sums and Gaussian vectors, Lecture Notes in Mathematics, Springer Verlag, Berlin, Heidelberg, Vol. $1617,1995$. 